\title{
Evaluation of a Hypertensive Rat Model Using Peripheral Blood Neutrophil Activity, Phagocytic Activity and Oxidized LDL Evaluation
}

\author{
RAN ZHANG ${ }^{1}$, HIROYUKI INAGAWA $^{1,2}$, KIMIKO KAZUMURA $^{3}$, HIROSHI TSUCHIYA $^{3}$, \\ TOSHIYUKI MIWA ${ }^{3}$, NAOKAZU MORISHITA ${ }^{4}$, SACHIKO UCHIBORI ${ }^{1}$, JUN HANASHIRO ${ }^{1}$, \\ TSUTOMU MASAKI ${ }^{5}$, HIDEKI KOBARA ${ }^{5}$ and GEN-ICHIRO SOMA ${ }^{1,2}$ \\ ${ }^{1}$ Control of Innate Immunity, Technology Research Association, Kagawa, Japan; \\ ${ }^{2}$ Research Institute for Healthy Living, Niigata University of Pharmacy and Applied Life Sciences, Niigata, Japan; \\ ${ }^{3}$ Central Research Laboratory, Hamamatsu Photonics K.K., Hamamatsu, Japan; \\ ${ }^{4}$ Electron Tube Division, Hamamatsu Photonics K.K., Hamamatsu, Japan; \\ ${ }^{5}$ Department of Gastroenterology and Neurology, Faculty of Medicine, Kagawa University, Kagawa, Japan
}

\begin{abstract}
Background/Aim: A system is being developed that can be used to easily evaluate the health condition of an individual with the help of trace amounts of a blood sample by focusing on xenobiotics. The system is called "Multimodal homeostasis evaluation system" (measurement of neutrophil activity, phagocytic activity of phagocytes and quantification of oxidized LDL (OxLDL)). To elucidate the possibility of using this system as an evaluation system for the health condition of an individual, clearly explaining the changes in various diseases is essential. In this study, evaluations were carried out using hypertensive model animals. Materials and Methods: Spontaneously hypertensive model rats SHR/NCrlCrlj and control rats WKY/NCrlCrlj were raised for 10 weeks from 6 to 16 weeks of age and their blood pressure was measured over time. Blood neutrophil activity (superoxide anion $\left(\mathrm{O}_{2}{ }^{-}\right)$generation and myeloperoxidase (MPO) activity) and phagocytic activity of phagocytes was measured by our developed apparatus (a simple prototype device under development). OxLDL was measured by an ELISA kit, and biochemical markers were measured using the blood sample. Results: Compared to WKY rats of the control group, systolic blood pressure, diastolic blood pressure, and mean blood pressure of SHR rats increased significantly with age. In SHR rats, there was a significant elevation in $\mathrm{O}_{2}{ }^{-}$
\end{abstract}

Correspondence to: Ran Zhang, Ph.D., Control of Innate Immunity, Technology Research Association, 2217-16 FROM-KAGAWA Bio Lab., Hayashi-cho, Takamatsu-shi, Kagawa-ken, 761-0301, Japan. Tel/Fax: +81 878139203, e-mail: zhang@shizenmeneki.org

Key Words: Peripheral blood, neutrophil activity, phagocytic activity, oxidized LDL. generation and MPO activity of neutrophils, alanine aminotransferase and triglycerides of blood, while phagocytic activity, OxLDL, low-density lipoprotein cholesterol, highdensity lipoprotein cholesterol, total cholesterol and totalbilirubin decreased. Conclusion: In the hypertensive model, biochemical markers were found to have a relationship with $\mathrm{O}_{2}{ }^{-}$generation, MPO activity, phagocytic activity of phagocytes, and OxLDL. This system is expected to be useful for clinical monitoring of hypertension diseases.

In recent years, the onset of chronic diseases, such as hypertension, arteriosclerosis, diabetes, and Alzheimer's disease are increasing due to lifestyle habits such as overeating, lack of exercise, and increased stress (1). It is considered that one of the onset mechanisms of lifestyle diseases is chronic inflammation caused due to the accumulation of xenobiotics such as viruses and bacteria, dead cells, denatured proteins, advanced glycation end products (AGEs), oxidized LDL (OxLDL), and amyloid- $\beta$ within an organism (2). The following three factors are considered important to evaluate the health condition of an individual based on the accumulation of xenobiotics. 1. Oxidative stress participates in the production of xenobiotics such as OxLDL and AGEs (3-4). It is considered that measurement of neutrophil activity can be one of the methods to evaluate the status of oxidative stress that increases with aging and stress (5). 2. Decrease in the ability of phagocytes increases the accumulation of foreign substances. It is considered that the ability of phagocytes to eliminate xenobiotics can be evaluated through phagocytic activity (6). 3. OxLDL has high cytotoxicity, and accumulation of OxLDL induces chronic inflammation (7). Therefore, OxLDL is considered as one of the representative 
molecules of xenobiotics, and quantification of OxLDL is useful in evaluating the accumulation status of xenobiotics.

Evaluating the health condition of an individual based on the above three aspects has so far presented problems such as the volume of a blood sample required is high, expensive, long evaluation time, and requirement of specialized personnel for measurement. For this reason, Hamamatsu Photonics K.K. (Shizuoka, Japan) developed a technology that simultaneously converts neutrophil activity into two types of optical information.The superoxide $\left(\mathrm{O}_{2}{ }^{-}\right)$production and myeloperoxidase (MPO) activity after stimulation with phorbol 12-myristate 13-acetate (PMA) in the hemolyzed blood sample was simultaneously determined with a real-time chemiluminescence and fluorescence monitoring system, comprised of a high speed on/off system with light emitting diode (LED) excitation light and a chemiluminescence/ fluorescence high sensitivity detector system (8). The instrument that measures the neutrophil activity can easily detect trace amounts of fluorescence with high sensitivity without hemolyzing erythrocytes (9). Making use of the fact that $\mathrm{pH}$-sensitive particles cause $\mathrm{pH}$ to be reduced in phagocytes along with increased fluorescence intensity, an improved instrument to measure the fluorescence intensity quickly was developed (Prototype) (FL- T1200; Hamamatsu Photonics K.K., Shizuoka, Japan). In addition, an improved instrument was developed so that fluorescence intensity can be measured conveniently, utilizing the fact that fluorescence intensity increases as shedding particles are reduced inside the phagocytes. The establishment of a method to conveniently evaluate the mouse phagocytic activity of macrophage strain J744.1 and trace human peripheral blood using the improved instrument was reported (10-11). In addition, a system has been developed to detect OxLDL using silkworm-type biotinylated lectin-like OxLDL receptor-1 ligand recognition region (12).

Our group carried out a study using arteriosclerosis model mice to examine the usefulness of the three aspects that focus on xenobiotics (13). Hypertension, a lifestyle disease was used in this study. The incidence of hypertension is high, and hypertension is known to be associated with the onset of stroke and cardiovascular diseases (14). On the other hand, there are many people who maintain their health despite being diagnosed as suffering from hypertension. Many people cannot be directly connected as having hypertension diseases despite suffering from hypertension. The usefulness of these three viewpoints were verified using spontaneously hypertensive model rats considering that if the indices evaluating the health condition changes, then the indices can be useful in finding a method to cope with hypertension.

\section{Materials and Methods}

Animals and treatments. Male 6-week-old SHR/Crij (Spontaneously Hypertensive Rat) rats and WKY/Crlj were obtained from Charles
River Laboratories Japan (Yokohama, Japan). The care and handling of the animals were in accordance with the Guidelines for the Care and Use of Laboratory Animals at Kagawa University and were approved by the Kagawa University Institutional Animal Care and Use Committee. The rats were housed under conditions of controlled temperature and humidity with a 12-h light/dark cycle and unrestricted access to food and water. A low-fat diet (LFD; $16.1 \mathrm{~kJ} / \mathrm{g}, 10 \%$ of energy as fat, D12450B) was purchased from Research Diets, Inc. (New Brunswick, NJ, USA).

After 1 week of prefeeding, the rats were divided into the following groups ( $\mathrm{n}=3$ per group): WKY group and SHR group. Each rat was then fed a low-fat diet for 10 weeks. Systolic blood pressure (SBP), diastolic blood pressure (DBP), and mean blood pressure (MBP) were monitored by tail-cuff methods (BP-98A, Softron Co., Tokyo, Japan) at 6 weeks old, 10 weeks old, 13 weeks old, and 16 weeks old. At 6 weeks old and 16 weeks old, blood samples were collected for $\mathrm{O}_{2}{ }^{-}$production and MPO activity from the tail vein. At 16 weeks old, plasma was collected from the heart and obtained by centrifugation. All surgeries were performed under diethyl ether anesthesia, and rats were euthanized by diethyl ether inhalation.

Measurement of biochemical markers. The level of plasma biochemical markers (triglyceride (TG), total cholesterol (T-CHO), low density lipoprotein cholesterol (LDL-C), high density lipoprotein cholesterol (HDL-C), aspartate transaminase (AST), alanine aminotransferase (ALT) and total-bilirubin (T-BIL)) were measured by Oriental Yeast Co. Ltd.

Simultaneous measurement of $\mathrm{O}_{2}{ }^{\cdot-}$ production and $\mathrm{MPO}$ activity by real-time monitoring of chemiluminescence and fluorescence $(C F L$ P2200; Hamamatsu Photonics K.K., Shizuoka, Japan). Thirty $\mu \mathrm{l}$ of peripheral blood was hemolyzed with hemolysis buffer (RBC Lysis Buffer, Bay bioscience, Kobe, Japan), and the cell sample was collected. Cell pellet was suspended in Ringer-Hepes buffer (154 mM $\mathrm{NaCl}, 5.6 \mathrm{mM} \mathrm{KCl}$, and $10 \mathrm{mM}$ Hepes, $\mathrm{pH}$ 7.4) and pre-incubated with $1 \mathrm{mM} \mathrm{CaCl} 2,0.5 \mu \mathrm{M}$ MCLA (6-(4-methoxyphenyl)-2-methyl3,7-dihydroimidazo[1,2-a]pyrazin-3-one hydrochloride; Tokyo Chemical Industry Co., Ltd., Tokyo, Japan), and $10 \mu \mathrm{M}$ aminophenyl fluorescein (APF; Goryo Chemical, Inc., Hokkaido, Japan) on a glass slide (total volume: $500 \mu \mathrm{l}$ ) for $2 \mathrm{~min}$ at $37^{\circ} \mathrm{C}$. Here, MCLA was used as an $\mathrm{O}_{2}{ }^{--}$-sensitive chemiluminescence probe and APF was used as a hypochlorous acid-sensitive fluorescence probe. The chemiluminescence intensity was calculated as relative chemiluminescence units (RCU), and the fluorescence intensity as relative fluorescence units (RFU) at a sampling rate of $(0.5 \mathrm{sec})$. The sample was continuously stirred at $37^{\circ} \mathrm{C}$ during the measurements. After $150 \mathrm{sec}$, PMA $(1 \mu \mathrm{M})$ was injected into the sample by an auto injector. The integration fluorescence intensity (iFI) and integration chemiluminescence intensity (iCI) were calculated as the peak intensity minus the basal value after PMA-induced responses.

Evaluation of phagocytic activity of rat peripheral blood using the Prototype (FL-T1200; Hmamastu Photonics K.K). A $1500 \mu 1$ phosphate-buffered saline (Sigma-Aldrich, St.Loius, MO, USA) containing $30 \mu \mathrm{g}$ of $\mathrm{pH}$-sensitive fluorescent particles (pHrodo, Green- Escherichia coli; Molecular probes, Carlsbad, CA, USA) was heated to $37^{\circ} \mathrm{C}$ with a block heater. During the measurement, $10 \mu \mathrm{l}$ of rat peripheral blood was added and the fluorescence intensity was measured immediately with the Prototype for 50 counts $(0.5 \mathrm{sec} / \mathrm{count})$. These values were defined as the negative 
control (the values without phagocytosis). After phagocytosis for 2 $\mathrm{h}$ at $37^{\circ} \mathrm{C}$, the fluorescence intensity was again measured with the Prototype for 50 counts $(0.5 \mathrm{sec} /$ count $)$. The difference in the fluorescence intensity obtained by subtracting the negative control value from the second measurements were defined as the phagocytic fluorescence intensity (PFI).

Measurement of plasma OxLDL. The levels of plasma OxLDL were measured using an ELISA kit (Kamiya Biomedical Company, Seattle, WA, USA), according to the manufacturer's instructions.

Statistical analysis. All results are expressed as the means \pm SEM. If the difference based on $t$-test or two-way analysis of variance (ANOVA) was significant, Tukey's multiple comparison test was used for paired comparisons using GraphPad Prism for Windows (GraphPad Software, Inc., San Diego, CA, USA). The results were considered to be significantly different at $p<0.05$.

\section{Results}

Changes in blood pressure during 6 to 16 weeks. At 6 weeks old, SBP, DBP and MBP showed no differences between the SHR group and the WKY group. From 10 weeks old, SBP, DBP and MBP were significantly higher in the SHR group than in the WKY group (Figure 1A-C).

Analysis of Biochemical marker. The levels of plasma LDLC, HDL-C, T-CHO and T-BIL were significantly lower in the SHR group than in the WKY group (Figure 2A-D), and ALT and T-BIL were significantly higher in the SHR group than in the WKY group (Figure $2 \mathrm{E}$ and F), but ALT were no differences between the SHR group and the WKY group (Figure 2G).

Change of the blood PFI, $\mathrm{O}_{2}{ }^{--}$production and $\mathrm{MPO}$ activity and the plasma OxLDL. At 6 weeks old, both $\mathrm{O}_{2}^{\cdot}$ production and MPO activity were no different between the SHR group and the WKY group. However, at 16 weeks old, both $\mathrm{O}_{2}{ }^{-}$production and MPO activity were significantly higher in the SHR group than in the WKY group (Figure 3AB). At 6 weeks old, PFI were significantly higher in the SHR group than in the WKY group. However, at 16 weeks old, PFI was significantly lower in the SHR group than in the WKY group (Figure 3C). At 16 weeks old, OxLDL was significantly lower in the SHR group than in the WKY group (Figure 3D).

Correlation between $\mathrm{O}_{2}{ }^{--}$production and $\mathrm{MPO}$ activity, $\mathrm{PFI}$, $O x L D L$ and biochemical markers. The correlation coefficient showing the correlation between the four indices and each biochemical marker is summarized in Table I. $\mathrm{O}_{2}{ }^{-}$ production showed a positive correlation with SBP (Pearson's correlation coefficient $(\mathrm{r}=0.270)$, ALT $(\mathrm{r}=0.717)$, AST $(\mathrm{r}=0.122)$ and $\mathrm{TG}(\mathrm{r}=0.672)$, and a negative correlation with T-CHO (r=-0.699), LDL-C ( $\mathrm{r}=-0.564)$, HDL-C $(\mathrm{r}=-0.649)$

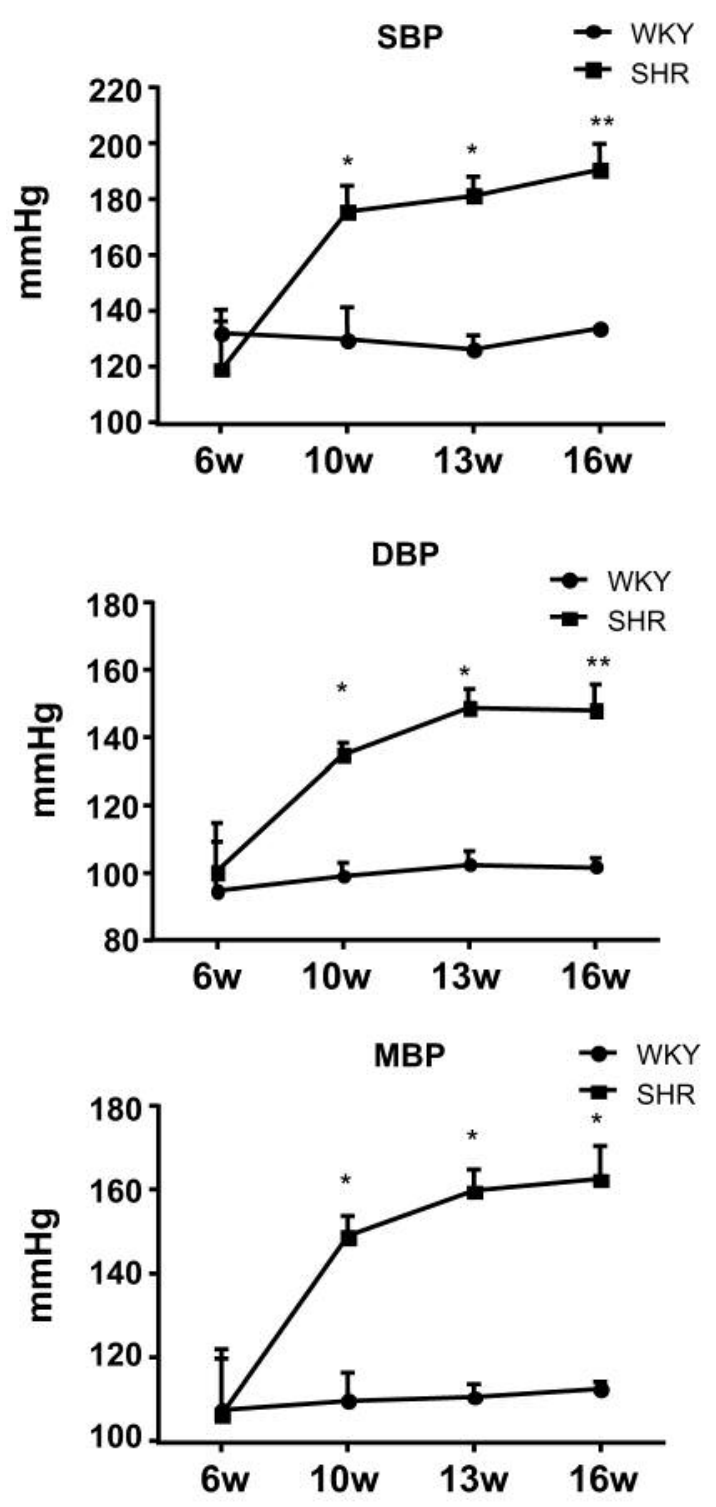

Figure 1. Changes in blood pressure over time between the SHR group and WKY group. Systolic blood pressure (SBP) (A), diastolic blood pressure $(D B P)(B)$, and mean blood pressure $(M B P)(C)$ were measured at 6 weeks old, 10 weeks old, 13 weeks old and 16 weeks old rats. Black circle: WKY group; black square: SHR group. The data are presented as the means \pm SEM and were obtained from 3 rats per group. $* p<0.05, * * p<0.01, * * * p<0.001$ compared to the WKY group.

and T-BIL ( $\mathrm{r}=-0.058)$. MPO activity showed a positive correlation with SBP $(r=0.859)$, ALT $(r=0.656)$ and AST $(\mathrm{r}=0.351)$ and a negative correlation with AST $(\mathrm{r}=-0.287), \mathrm{T}-$ $\mathrm{CHO}(\mathrm{r}=-0.705)$, LDL-C ( $\mathrm{r}=-0.762)$, HDL-C $(\mathrm{r}=-0.715)$ and T-BIL ( $r=-0.776)$. PFI showed a positive correlation with SBP ( $r=0.429)$, T-CHO ( $r=0.841)$, LDL-C ( $r=0.738)$, HDL-C $(\mathrm{r}=0.775)$ and T-BIL $(\mathrm{r}=0.675)$, and a negative correlation with ALT $(r=-0.879)$, AST $(r=-0.059)$ and TG $(r=-0.784)$. 

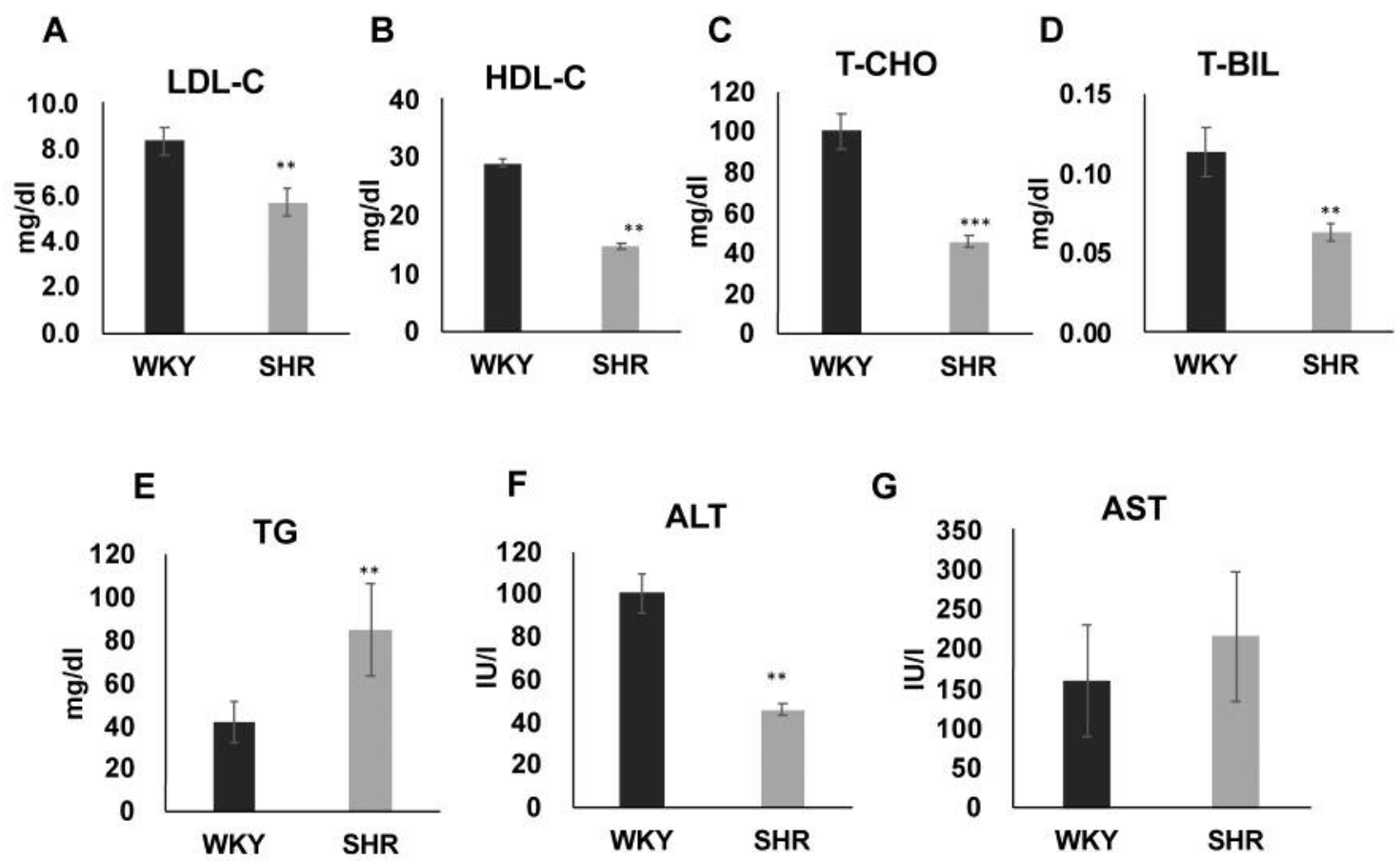

Figure 2. The level of plasma biochemical markers between the SHR and WKY group. LDL-C: Low density lipoprotein cholesterol (A), HDL-C: high density lipoprotein cholesterol (B), T-CHO: total cholesterol (C), TG: triglyceride (D), ALT: alanine aminotransferase (E), T-BIL: total bilirubin $(F)$. Black column: WKY group; gray column: SHR group. The data are presented as the means \pm SEM and were obtained from 3 rats per group. $* * p<0.01, * * * p<0.001$ compared to the WKY group.

OxLDL showed a positive correlation with AST ( $\mathrm{r}=0.025)$, TCHO (r=0.745), LDL-C ( $\mathrm{r}=0.737)$, HDL-C ( $\mathrm{r}=0.630)$ and $\mathrm{T}-$ $\mathrm{BIL}(\mathrm{r}=0.592)$, and a negative correlation with SBP $(\mathrm{r}=-0.756)$, $\operatorname{ALT}(\mathrm{r}=-0.794)$ and TG (r=-0.691).

\section{Discussion}

Hypertension, diabetes, aging, dyslipidemia have been epidemiologically proven to be risk factors for stroke and cardiovascular diseases (15-17). Commonly used SHR hypertensive model rats are also known to have lipid metabolism abnormality along with spontaneous hypertension (18). In this study, it was confirmed that, SBP, DBP and MBP of SHR rats increase significantly with age (in weeks) when compared to the WKY group (Figure 1). In SHR rats, LDL$\mathrm{C}$, HDL-C, and T-CHO decreased significantly, while there was a significant increase in TG (Figure 2A-C and E). Hypertensive model rats have also been confirmed as having elevated blood pressure and lipid metabolism abnormality.

In this study, it was identified how the four indices (phagocytic activity, $\mathrm{O}_{2}{ }^{--}$generation amount, MPO activity, and OxLDL amount) obtained from the peripheral blood, change with the increase in SBP, DBP and MBP (Figure 3). Compared to the WKY group, the amount of $\mathrm{O}_{2}{ }^{--}$generation
Table I. Correlation coefficient list between 4 indicators $\left(\mathrm{O}_{2} \cdot-\right.$, $M P O$, $P F I$ and $O x L D L)$ and biochemical markers.

\begin{tabular}{lrrrc}
\hline $\mathrm{R}^{2}$ & $\mathrm{O}_{2}{ }^{-}(\mathrm{iCI})$ & MPO (iFI) & PFI & OxLDL (pg/ml) \\
\hline SBP (mmHg) & 0.270 & 0.859 & 0.429 & -0.756 \\
ALT (IU/l) & 0.717 & 0.656 & -0.879 & -0.794 \\
AST (IU/l) & 0.122 & -0.287 & -0.059 & 0.025 \\
T-CHO (mg/dl) & -0.699 & -0.705 & 0.841 & 0.745 \\
TG (mg/dl) & 0.672 & 0.351 & -0.784 & -0.691 \\
LDL-C (mg/dl) & -0.564 & -0.762 & 0.738 & 0.737 \\
HDL-C (mg/dl) & -0.649 & -0.715 & 0.775 & 0.630 \\
T-BIL (mg/dl) & -0.058 & -0.776 & 0.675 & 0.592 \\
\hline
\end{tabular}

and MPO activity significantly increased in SHR group, while phagocytic activity significantly decreased, and there was hardly any change in the amount of OxLDL produced. Hypertension in older people has been reported to correlate with 8-isoprostane, a type of oxidative stress marker. In this study, the neutrophil activity $\left(\mathrm{O}_{2}{ }^{--}\right.$production amount and MPO activity) which is considered an index of oxidative stress, was found to be elevated. It is thought that in high blood pressure state, foreign bodies in the living body tend 

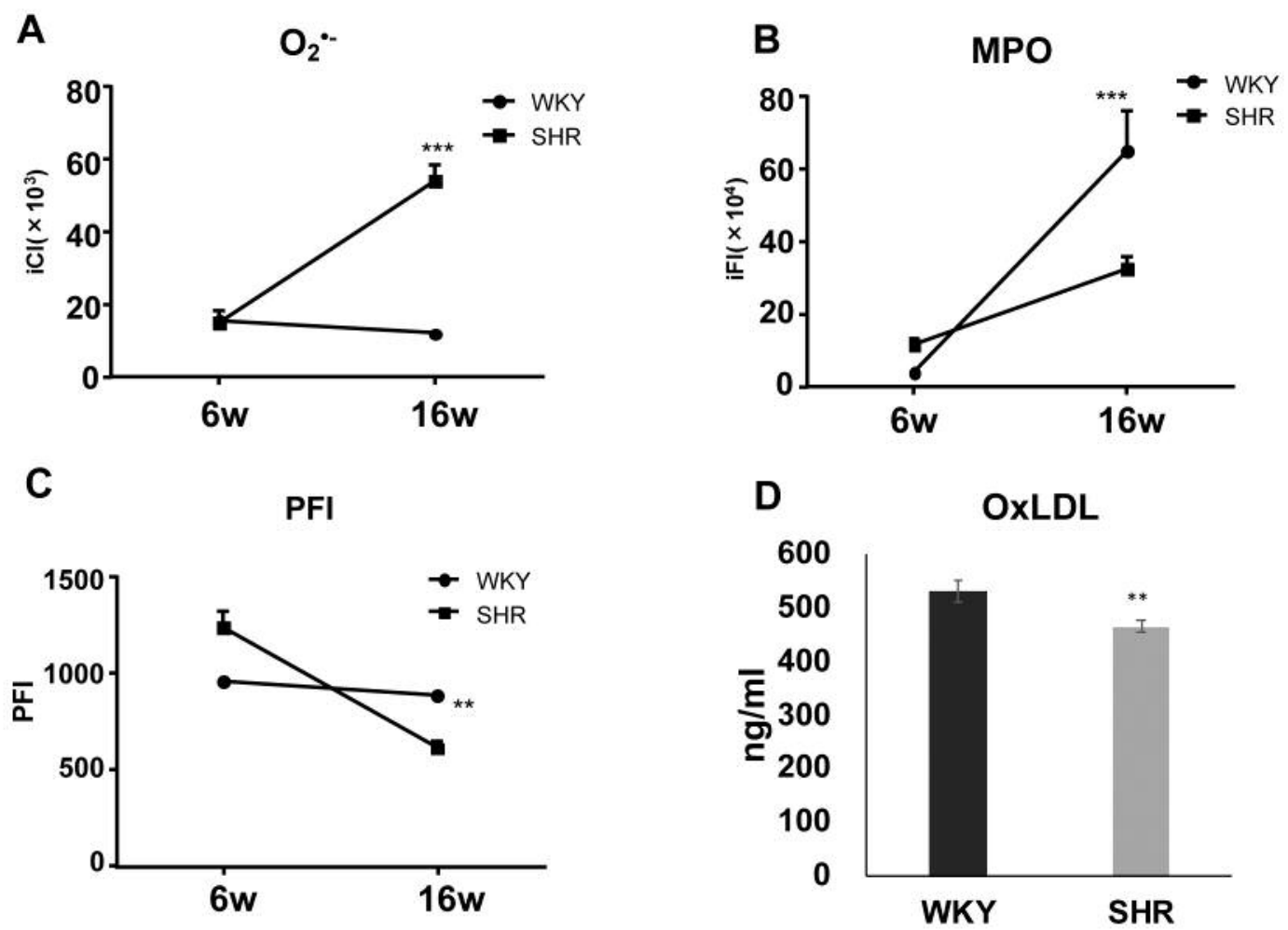

Figure 3. Changes in $\mathrm{O}_{2}^{\circ-}, \mathrm{MPO}, \mathrm{PFI}$, and $\mathrm{OxLDL} . \mathrm{O}_{2}^{-}$changes at 6 weeks old and 16 weeks old rats (A), MPO changes in at 6 weeks old and 16 weeks old rats (B), PFI changes at 6 weeks old and 16 weeks old rats $(C)$. Black circle: WKY group; black square: SHR group. OxLDL was measured at 16 weeks old rats (D). Black column: WKY group; gray column: SHR group. The data are presented as the means \pm SEM and were obtained from 3 rats per group. ${ }^{* *} p<0.01, * * * p<0.001$ compared to the WKY group.

to be formed, and oxidative stress is developed (19). Also, the decrease in the phagocytic activity in SHR rats of 16 weeks of age was due to deterioration in the ability to eliminate xenobiotics. There is no difference in the amount of OxLDL in rats with hypertension and rats that are normal, but OxLDL is not accumulated in the early stages of hypertension. As the duration of hypertension increases, there is a possibility that OxLDL gets accumulated in SHR rats. The periodical changes in OxLDL in animals raised over a long duration of time should be confirmed in the future.

When the usefulness of this evaluation system was verified in the past using arteriosclerosis model mice, the generation amount of $\mathrm{O}_{2}^{-}$generation, MPO activity, and OxLDL increased significantly in the arteriosclerosis model mice compared to the regular mice, while the phagocytic activity showed a slightly decreasing trend (13). The 4 indices indicated changes that were different from the hypertensive model. This is because, the indices are considered to indicate different dynamics according to the condition of each disease.
On the other hand, items of each of the four indices related to the general biochemical markers were identified to be different. For example, T-BIL did not show any correlation with $\mathrm{O}_{2}{ }^{\circ-}$ generation, but indicated a negative correlation with MPO activity. Further, the four indices were also observed to have a relationship with ALT, but not with AST. Each of these four indices can be considered as independent evaluation factors, and measuring each index of this evaluation system is meaningful.

If the health condition of an individual (state of oxidative stress, xenobiotic exclusion ability, and xenobiotic accumulation status) is evaluated using this system, then the indices can be expected to become new indices for the treatment policy of patients with various diseases such as cancer. This is a convenient system that is minimally invasive with fewer ethical problems, and causes less burden on the patients.

\section{Conflicts of Interest}

The Authors have no financial conflicts of interest. 


\section{Acknowledgements}

This work was partly supported by the Council for Science, Technology and Innovation (CSTI), Cross-ministerial Strategic Innovation Promotion Program (SIP), "Technologies for creating nextgeneration agriculture, forestry and fisheries" (funding agency: Biooriented Technology Research Advancement Institution, NARO).

\section{References}

1 Vallgårda S: Why the concept "lifestyle diseases" should be avoided. Scand J Public Health 39(7): 773-775, 2011.

2 Belloc NB and Breslow J: Relationship of physical health status and health practices. Prew Med 1(3): 409-421, 1972.

3 Halliwell B and Gutteridge JMC: Free Radicals in Biology and Medicine. 4th ed., New York, Oxford University Press, 2007.

4 Vitte J, Michel BF, Bongrand P and Gastaut JL: Oxidative stress level in circulating neutrophils is linked to neurodegenerative diseases. J Clin Immunol 24(6): 683-692, 2004.

5 Breitmeier D, Becker N, Weilbach C, Albrecht K, Scheinichen D, Panning B, Schneider U and Jüttner B: Ethanol-induced malfunction of neutrophils respiratory burst on patients suffering from alcohol dependence. Alcohol Clin Exp Res 32(10): 17081713,2008

6 Lecube A, Pachon G, Petriz J, Hernandez C and Simo R: Phagocytic activity impaired in type 2 diabetes mellitus and increases after metabolic imprevement. Plos ONE 6: e23360, 2011.

7 Sata $\mathrm{M}$ and Walsh K: Oxidized LDL actibates fas-mediated endothelial cell apoptosis. J Clin Invest 102(9): 1682-1689, 1998.

8 Kazumura K, Sato Y, Satozono H, Koike T, Tsuchiya H, Hiramatsu M, Katsumata $M$ and Okazaki S: Simultaneous monitoring of superoxides and intracellular calcium ions in neutrophils by chemiluminescence and fluorescence: Evaluation of action mechanisms of bioactive compounds in foods. J Pharm Biomed Anal 84: 90-96, 2013.

9 Kazumura K, Tsuchiya H and Osawa T: Evaluate the activity of neutrophil cells method. Patent 2015-84757 (P2015-84757A), 2015.

10 Zhang R, Kobayashi Y, Kazumura K, Tsuchiya H, Morishita N, Inagawa $\mathrm{H}$ and Soma G: Development of an evaluation device for phagocytic activity of new phagocytes using simple and $\mathrm{pH}$ sensitive particles that do not require pre-treatment. Anticancer Res 36: 3613-3618, 2016.
11 Zhang R, Inagawa H, Takahashi M, Kawanishi H, Kazumura K, Tsuchiya H, Morishita N, Kobayashi Y, Masaki T, Kobara H and Soma G: Measurement of the phagocytic activity of human peripheral blood using a highly sensitive fluorometric detection device without hemolysis. Anticancer Res 37: 3897-3903, 2017.

12 Kumano-Kuramochi M, Shimozu Y, Wakita C, OhnishiKameyama M, Shibata T, Matsunaga S, Takano-Ishikawa Y, Watanabe J, Goto M, Xie Q, Komba S, Uchida K and Machida $\mathrm{S}$ : Identification of 4-hydroxy-2-nonenal-histidine adducts that serve as ligands for human lectin-like oxidized LDL receptor-1. Biochem J 442(1): 171-180, 2012.

13 Kobayashi Y, Inagawa H, Kohchi C, Kazumura K, Tsuchiya H, Miwa T, Okazaki K and Soma G: Oral administration of Pantoea agglomerans- derived lipopolysaccharide prevents development of atherosclerosis in high-fat diet-fed apoE-deficient mice via ameliorating hyperlipidemia, pro-inflammatory mediators and oxidative responses. PLOS ONE 13(3): e0195008, 2018.

14 Higashi Y, Sasaki S, Kurisu S Yochimizu A, Sasaki N, Matsuura $\mathrm{H}$, Kajiyama $\mathrm{G}$ and Oshima T: Regular aerobic exercise augments endothelium-dependent vascular relaxation in normotensive as well as hypertensive subjects - role of endothelium-derived nitric oxide. Circulation 100(11): 1194-1202, 1999.

15 Hopkins PN, Hunt SC, Wu LL, Williams GH and Williams RR: Hypertension, dyslipidemia, and insulin resistance: links in a chain or spokes on a wheel? Curr Opin Lipidol 7(4): 241-253, 1996.

16 Susic D: Hypertension, aging, and atherosclerosis. The endothelial interface. Med Clin North Am 81(5): 1231-1240, 1997.

17 Grossman E and Messerli FH: Hypertension and diabetes. Adv Cardiol 45: 82-106, 2008.

18 Hajri T, Ibrahimi A, Coburn CT, Knapp FF Jr, Kurtz T, Pravenec $\mathrm{M}$ and Abumrad NA: Defective fatty acid uptake in the spontaneously hypertensive rat is a primary determinant of altered glucose metabolism, hyperinsulinemia, and myocardial hypertrophy. J boil Chem 276(26): 23661-23666, 2001.

19 Dohi Y, Ohashi M, Sugiyama M, Takase H, Sato K and Ueda R: Candesartan reduces oxidative stress and inflammation in patients with essential hypertension. Hypertens Res 26(9): 691697, 2003. 